\title{
Atendimento e manejo de pacientes queimados: Revisão integrativa
}

\author{
Care and management of burned patients: Integrative review \\ Cuidado y manejo de pacientes quemados: Revisión integrativa
}

Recebido:19/05/2021 | Revisado: 28/05/2021 | Aceito: 29/05/2021 | Publicado: 11/06/2021

Igor dos Santos Cavalcante

ORCID: https://orcid.org/0000-0003-2907-9026

Universidade Federal do Delta do Parnaíba, Brasil E-mail: igorsc@live.com

Maria Simone Lopes

ORCID: https://orcid.org/0000-0003-2689-3655 Universidade Federal do Delta do Parnaíba, Brasil E-mail: mariasimonemed@ufpi.edu.br

João Pedro Sousa Mendes

ORCID: https://orcid.org/0000-0002-0721-2845

Universidade Federal do Delta do Parnaíba, Brasil E-mail: mendespedro00@gmail.com

Lucas de Carvalho Techi

ORCID: https://orcid.org/0000-0002-8226-7774

Universidade Federal do Delta do Parnaíba, Brasil

E-mail: lucascarvalhotechi@hotmail.com

Danilo Andrade Lima

ORCID: https://orcid.org/0000-0003-3910-319X

Universidade Federal do Delta do Parnaíba, Brasil E-mail: danilo_al@live.com

Jocerone Emerson Nogueira Oliveira

ORCID: https://orcid.org/0000-0002-6025-3929

Universidade Federal do Delta do Parnaíba, Brasil E-mail: joceroneemerson1@gmail.com

Danielle Laís Lopes Barboza

ORCID: https://orcid.org/0000-0002-6237-2163

Universidade Federal do Delta do Parnaíba, Brasil E-mail: daniellelaislopes@gmail.com

Ytallo Samuel Oliveira Barros

ORCID: https://orcid.org/0000-0002-5495-7348

Universidade Federal do Rio Grande do Norte, Brasil

E-mail: ytallobarros@hotmail.com

Ludmilla Figueiredo do Vale Fontenelle

ORCID: https://orcid.org/0000-0003-4885-8340

Universidade Federal do Delta do Parnaíba, Brasil

E-mail: lufigueiredo@hotmail.com

Lúcia Maria de Sousa Aguiar dos Santos

ORCID: ttps://orcid.org/0000-0002-9316-2214 Universidade Federal do Piauí, Brasil E-mail: 1msasantos@gmail.com

Pedro Marcos Gomes Teixeira

ORCID: https://orcid.org/0000-0003-1779-3222

Uninovafapi, Brasil

E-mail: pedromgt@gmail.com

\begin{abstract}
Resumo
Objetivo: descrever as condutas adequadas para o manejo de pacientes queimados, de acordo com o nível de assistência (pré-hospitalar ou hospitalar), a gravidade e as particularidades no atendimento. Metodologia: Trata-se de uma revisão integrativa de literatura de artigos completos disponíveis nas bases de dados LILACS, SciELO e MEDLINE, utilizando como descritores "Queimaduras", "Unidades de Queimados" e "Primeiros socorros", publicados entre os anos de 2010 e 2020 . Após análise crítica, foram selecionados 8 artigos de acordo com os critérios de inclusão, que compuseram a amostra final do estudo. Resultados: O atendimento adequado ao paciente vítima de queimadura deve seguir protocolos rígidos e pré-estabelecidos, destacando-se os cuidados pré-hospitalares com a lesão, de acordo com o tipo de queimadura ocorrida, assim como deve prosseguir com o tratamento de uma equipe multiprofissional, respeitando-se as particularidades na conduta dos casos de populações especiais. Conclusão: $\mathrm{O}$ manejo de pacientes queimados exige habilidades, conhecimentos e avaliação clínica específicos para a evolução de melhor prognóstico.
\end{abstract}


Palavras-chave: Queimaduras; Unidades de queimados; Primeiros socorros.

\begin{abstract}
Objective: to describe the appropriate conducts for the management of burn patients, according to the level of assistance (pre-hospital or hospital), the severity and the particularities in the care. Methodology: This is an integrative literature review of complete articles available in the LILACS, SciELO and MEDLINE databases, using as descriptors "Burns", "Burn Units" and "First Aid", published between 2010 and 2020. After a critical analysis, 8 articles were selected according to the inclusion criteria, which comprised the final sample of the study. Results: Adequate care for burn victim patients must follow rigid and pre-established protocols, highlighting pre-hospital care with the injury, according to the type of burn that occurred, as well as continuing with the treatment of a team multiprofessional, respecting the particularities in the conduct of cases of special populations. Conclusion: The management of burn patients requires specific skills, knowledge and clinical assessment for the evolution of a better prognosis.
\end{abstract}

Keywords: Burns; Burn units; First aid.

\title{
Resumen
}

Objetivo: describir las conductas adecuadas para el manejo del paciente quemado, según el nivel de asistencia (prehospitalaria u hospitalaria), la gravedad y las particularidades en la atención. Metodología: Se trata de una revisión bibliográfica integradora de artículos completos disponibles en las bases de datos LILACS, SciELO y MEDLINE, utilizando como descriptores "Quemaduras", "Unidades de Quemaduras" y "Primeros Auxilios", publicados entre 2010 y 2020. Luego de un análisis crítico, se seleccionaron 8 artículos de acuerdo a los criterios de inclusión, que conformaron la muestra final del estudio. Resultados: La atención adecuada a los pacientes quemados debe seguir protocolos rígidos y preestablecidos, destacando la atención prehospitalaria con la lesión, según el tipo de quemadura ocurrida, así como continuar con el tratamiento de un equipo. multiprofesional, respetando las particularidades en la conducción de casos de poblaciones especiales. Conclusión: El manejo del paciente quemado requiere de habilidades, conocimientos y valoración clínica específicos para la evolución de un mejor pronóstico.

Palabras clave: Quemaduras; Unidades de quemados; Primeros auxilios.

\section{Introdução}

As queimaduras são lesões teciduais comuns e evitáveis, decorrentes de trauma térmico, elétrico, biológico, químico ou radioativo, que destrói parcial ou totalmente a pele e seus anexos, podendo alcançar camadas mais profundas como o tecido celular subcutâneo, músculos, tendões e ossos. Dependendo da natureza e da gravidade da lesão, o paciente pode sofrer sequelas irreversíveis, sofrimento físico e psicológico e evoluir para óbito (Lima, et al., 2017; Rocha, Silva, Silva, Melo, \& Mota, 2020).

As queimaduras constituem um grave problema de saúde pública no Brasil uma vez que representam uma das principais causas de atendimentos nos serviços de urgência e emergência, além das repercussões físicas, sociais e econômicas (Bezerra et al., 2020; Moulin et al., 2018). Estima-se que aconteçam aproximadamente 1.000 .000 acidentes com queimaduras por ano, destes, 100.000 pacientes irão procurar atendimento e 2.500 irão a óbito em decorrência das lesões e das suas complicações. Sabe-se, ainda, que a maioria das vítimas é do sexo masculino, predominantemente adultos jovens entre 20 e 29 anos e crianças menores de 10 anos (Brasil, 2017).

As lesões podem ser categorizadas quanto à sua etiologia, extensão, profundidade, região do corpo atingida e tempo de exposição. A avaliação destes fatores são essenciais na definição da gravidade, na escolha da terapêutica mais adequada, no início precoce dos cuidados, interferindo diretamente no prognóstico do paciente e na redução do número de óbitos (Cantarellikantorski et al., 2014).

Nessa perspectiva, consegue-se ainda destacar a importância do cuidado multidisciplinar frente a pacientes tão complexos como os queimados, já que estes devem se tratados de forma holística desde sua entrada até sua reabilitação e posterior reintegração social (Faroni et al., 2018).

Sendo assim, fica exposta a relevância da adequada assistência multidisciplinar para os pacientes acometidos por este tipo de trauma, sendo essencial o estabelecimento e padronização das condutas adotadas pela equipe assistencial, além do 
desenvolvimento de políticas públicas de prevenção e tratamento de queimaduras, sendo a estratégia chave para redução da morbidade, mortalidade e custos relacionados a este trauma (Moraes et al., 2016; Gilbert et al., 2017). Portanto, o objetivo desse trabalho consiste em descrever as condutas adequadas para o manejo de pacientes queimados, de acordo com o nível de assistência (pré-hospitalar ou hospitalar), a gravidade e as particularidades no atendimento.

\section{Metodologia}

Trata-se de uma revisão integrativa, que visa analisar e sintetizar o que a literatura traz sobre a assistência e o manejo do paciente queimado, abordando as particularidades do atendimento inicial e primeiros-socorros dos casos de queimaduras, sistematizando as práticas adotadas, com foco na evolução da prática clínica e potencialização da tomada de decisão.

A abordagem adotada no estudo foi quantitativa, levando-se em consideração os fundamentos de uma revisão de literatura, os quais consistem em: (1) identificação do tema e seleção da questão norteadora; (2) definição de critérios de inclusão e exclusão de estudos; (3) investigação na literatura; (4) estabelecimento das informações a serem coletadas dos trabalhos selecionados; (5) análise dos estudos selecionados; (6) interpretação dos resultados e (7) síntese das informações.

A busca de artigos foi feita nas seguintes bases de dados: Scientific Eletronic Library Online (SciELO), Medical Literature Analysis and Retrieval System Online (Medline) e Literatura Latino-Americana e do Caribe (LILACS), utilizandose os Descritores em Ciências das Saúde (DECs): "Queimaduras", "Unidades de queimados" e "Primeiros socorros".

Os critérios de inclusão foram: artigos científicos disponibilizados de forma integral, que estivessem enquadrados como pesquisas em seres humanos com ano de publicação entre 2010 e 2020. Os critérios de exclusão foram: materiais que não fossem artigos científicos e estudos que não estivessem de acordo com o tema selecionado.

Ao utilizar os três descritores associados na base LILACS, foram encontrados 2 artigos. Em seguida, a busca foi feita com os descritores em pares: com "Unidades de queimados" e "Primeiros socorros" foram encontrados 2 artigos; o par "queimaduras" e "unidades de queimados" apresentou 252 artigos; o par "queimaduras" e "primeiros socorros" teve 24 artigos encontrados.

Na base SciELO, ao utilizar os três descritores em associação não foram encontrados artigos; ao usar o par "queimaduras" e "primeiros socorros" foi encontrado apenas 1 artigo; utilizando o par "queimaduras" e "unidades de queimados" foram encontrados 16 artigos, o outro par não obteve resultados.

Na base MEDLINE, ao utilizar os três descritores foram encontrados 37 artigos; ao usar o par "queimaduras" e "primeiros socorros" foram encontrados 530 artigos; utilizando o par "unidades de queimados" e "primeiros socorros" foram encontrados 37; ao usar o par "queimaduras" e "unidades de queimados" foram encontrados 2.534 artigos.

No total, foram encontrados 3.435 artigos na busca inicial, dos quais foram descartados 2.018 artigos porque estavam incompletos nas bases pesquisadas. Assim, restaram 1.417 artigos para a leitura do título, dos quais foram descartados 1.397 artigos por não estarem diretamente relacionados ao estudo. A seguir, sobraram 20 artigos para leitura, excluindo-se 12 por não adequação ao foco da discussão. Por conseguinte, foram selecionados 8 artigos, descritos no Quadro 1, para a leitura integral para a composição final desse estudo. O fluxograma da Figura 1 detalha cada etapa da revisão de literatura realizada.

A pesquisa foi realizada em Maio de 2021 e os artigos escolhidos foram submetidos a instrumento no software Microsoft Excel 2016 contendo as variáveis: título do artigo, ano de publicação, periódico e objetivo do estudo. 
Figura 1 - Fluxograma detalhando cada etapa do processo de seleção de artigos para a revisão.
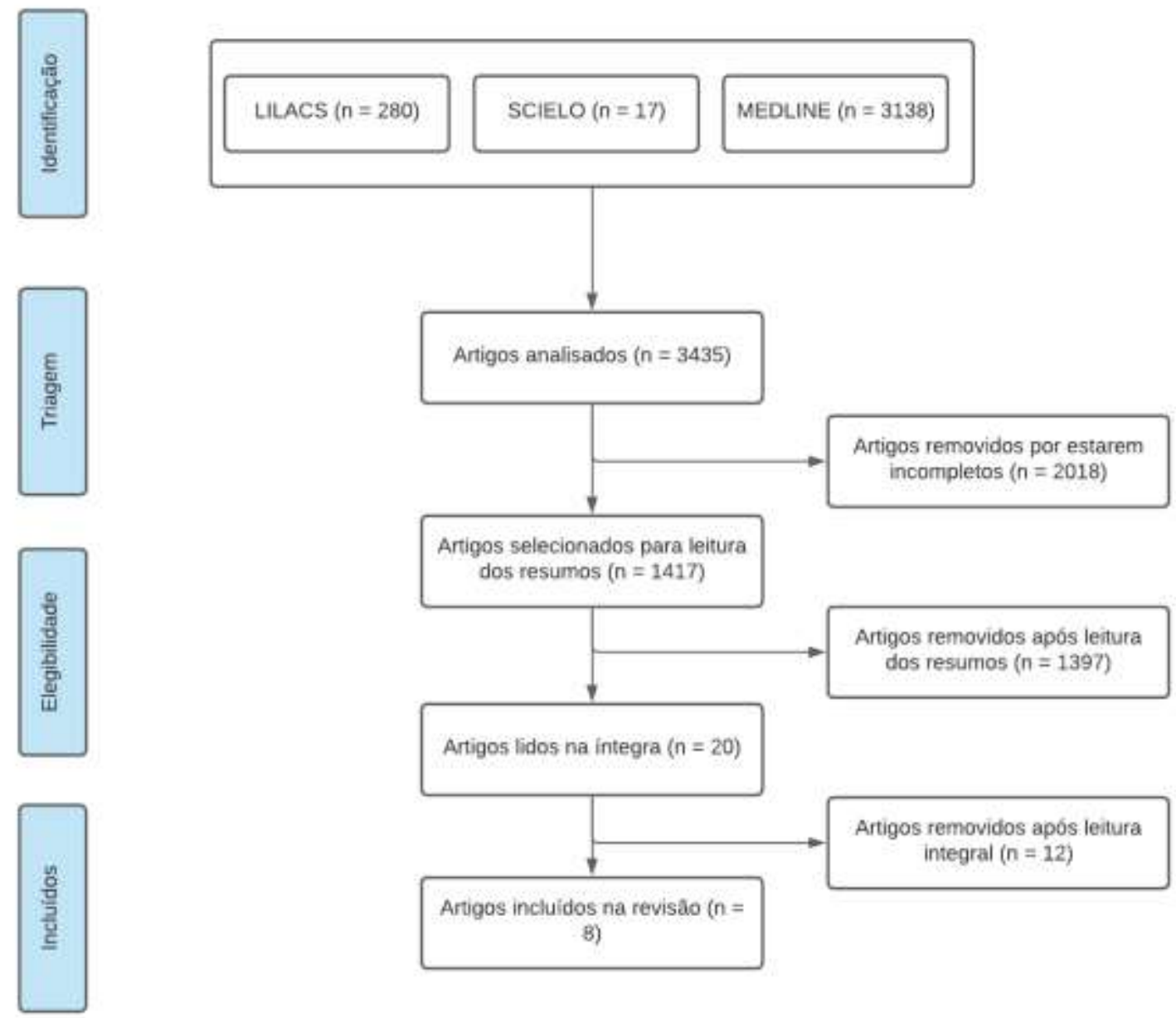

Fonte: Cavalcante et al (2021).

\section{Resultados}

Os artigos selecionados foram numerados de A1 a A8 e organizados em uma tabela para que seja exposto o seu título, periódico de publicação, ano e o objetivo do estudo, conforme consta no Quadro 1.

Há 1 artigo de 2019 (A5), 4 artigos de 2018 (A1, A3, A6, A8), 1 artigo de 2016 (A7), 1 artigo de 2014 (A2) e 1 artigo de 2012 (A4). Desse modo, com a intenção de realizar uma busca completa, os critérios de inclusão foram artigos dos últimos 10 anos relativos ao atendimento inicial do paciente queimado, contudo evitando-se referências ultrapassadas e sem relevância para a revisão.

O estudo A1 demonstra a importância que o atendimento pré-hospitalar eficiente exerce no prognóstico positivo de pacientes queimados, destacando a necessidade da lavagem correta da lesão e retirada de adornos das vítimas, como formas de diminuir possíveis complicações, além de relatar os procedimentos adequados que devem ser incorporados na conduta préhospitalar de qualidade.

No trabalho A2 é feita uma análise do impacto que a implantação de um protocolo específico no atendimento ao paciente queimado possui no manejo proficiente dos casos, relatando a indispensabilidade da existência de medidas padronizadas, através de protocolos assistenciais com recomendações técnicas e científicas, no intuito de tornar-se um instrumento seguro no acompanhamento e controle de casos. 
Já nos artigos A3, A4 e A5, estuda-se as particularidades no atendimento de casos específicos, como gestantes, pacientes pediátricos e pacientes com queimadura por eletricidade, respectivamente.

O artigo A6: vem trazendo a importância do atendimento interdisciplinar em pacientes queimados, considerados pacientes desafiadores a nível médico, psicológico e social, pois as queimaduras também abordam fatores sociais que o paciente possa vir sofrer e por isso justifica-se a necessidade de outros profissionais para acompanhamento como: psicológico e fisioterapeuta.

No artigo A7 aborda os tipos de queimaduras, e afirma a importância do primeiro atendimento e classificação para posterior manejo e tratamento adequado conforme a necessidade do paciente pediátrico, pacientes que possuem peculiaridades e fragilidades que necessitam de maiores cuidados e atenção.

O trabalho A8, afirma que as queimaduras constituem uma das principais causas de morbimortalidade em crianças nos primeiros anos de vida. O paciente pediátrico tem suas peculiaridades e, assim, o tratamento deve seguir conforme o grau de queimadura nesse paciente.

Quadro 1 - Distribuição dos resultados dos artigos encontradas na busca relacionados ao atendimento e manejo de pacientes queimados, de acordo com seu nome, periódico, ano e objetivo.

\begin{tabular}{|c|c|c|c|c|}
\hline OBRA & NOME DO ARTIGO & PERIÓDICO & ANO & OBJETIVO \\
\hline A1 & $\begin{array}{l}\text { Importância de um atendimento pré- } \\
\text { hospitalar efetivo a adultos vítimas } \\
\text { de queimaduras: uma revisão } \\
\text { integrativa. }\end{array}$ & $\begin{array}{l}\text { Revista Brasileira } \\
\text { de Queimaduras }\end{array}$ & 2018 & $\begin{array}{l}\text { Identificar e analisar artigos a respeito das condutas no } \\
\text { atendimento pré-hospitalar ao paciente queimado. }\end{array}$ \\
\hline $\mathrm{A} 2$ & $\begin{array}{l}\text { Análise comparativa } \\
\text { morbimortalidade antes e após } \\
\text { implantação de protocolo de } \\
\text { atendimento ao queimado. }\end{array}$ & $\begin{array}{l}\text { Revista Brasileira } \\
\text { de Queimaduras }\end{array}$ & 2014 & $\begin{array}{l}\text { Analisar a morbimortalidade de um hospital de urgência } \\
\text { antes e depois da implantação de um protocolo de } \\
\text { queimados. }\end{array}$ \\
\hline $\mathrm{A} 3$ & $\begin{array}{l}\text { Gestação e queimadura: experiência } \\
\text { de unidade. de queimaduras em } \\
\text { Hospital Universitário. }\end{array}$ & $\begin{array}{l}\text { Revista Brasileira } \\
\text { de Cirurgia } \\
\text { Plástica }\end{array}$ & 2018 & $\begin{array}{l}\text { Relatar a experiência no atendimento e manejo de duas } \\
\text { pacientes grávidas queimadas. }\end{array}$ \\
\hline A4 & $\begin{array}{l}\text { Medical management of paediatric } \\
\text { burn injuries: Best practice. }\end{array}$ & $\begin{array}{l}\text { Journal of } \\
\text { Paediatrics and } \\
\text { Child Health }\end{array}$ & 2012 & $\begin{array}{l}\text { Avaliar as particularidades no atendimento de pacientes } \\
\text { pediátricos que sofreram queimaduras. }\end{array}$ \\
\hline A5 & $\begin{array}{l}\text { Electrical Injuries of the Hand and } \\
\text { Upper Extremity. }\end{array}$ & $\begin{array}{l}\text { Journal of the } \\
\text { American } \\
\text { Academy of } \\
\text { Orthopaedic } \\
\text { Surgeons }\end{array}$ & 2019 & $\begin{array}{l}\text { Tratar das queimaduras por eletricidade, de acordo com } \\
\text { sua característica, fisiopatologia e atendimento } \\
\text { específico. }\end{array}$ \\
\hline
\end{tabular}




\begin{tabular}{|c|l|c|c|l|}
\hline A6 & $\begin{array}{l}\text { Manejo interdisciplinario de } \\
\text { pacientes quemados: pilar } \\
\text { fundamental para la reducción de } \\
\text { secuelas funcionales. }\end{array}$ & $\begin{array}{c}\text { Revista médica de } \\
\text { rosário }\end{array}$ & 2018 & $\begin{array}{l}\text { Demonstrar a eficácia de manejo interdisciplinar em } \\
\text { pacientes queimados } \\
\text { AB e B de zonas funcionais para reduzir sequelas. }\end{array}$ \\
\hline A7 & $\begin{array}{l}\text { Initial Assessment and Management } \\
\text { of Thermal Burn Injuries in Children. }\end{array}$ & $\begin{array}{c}\text { Pediatrics in } \\
\text { review }\end{array}$ & 2016 & $\begin{array}{l}\text { Descrever os tipos de queimaduras e a avaliação inicial } \\
\text { de uma criança queimada em termos de profundidade, } \\
\text { tamanho e lesões associadas ou condições médicas. } \\
\text { Descrever as queimaduras apropriadas para o tratamento } \\
\text { ambulatorial. Estimar os requisitos iniciais de } \\
\text { reanimação de líquidos nas primeiras 24 horas em } \\
\text { crianças com queimaduras extensas (> 25\% da superfície } \\
\text { corporal total) de espessura parcial. }\end{array}$ \\
\hline A8 & $\begin{array}{l}\text { Propuesta de tratamiento del gran } \\
\text { quemado en la unidad de cuidados } \\
\text { intensivos del CHPH. }\end{array}$ & $\begin{array}{l}\text { Arquivos de } \\
\text { pediatria do } \\
\text { Uruguai. }\end{array}$ & 2018 & $\begin{array}{l}\text { Guia prático abordando o retorno do paciente à unidade } \\
\text { de tratamento intensivo. }\end{array}$
\end{tabular}

Fonte: Cavalcante et al (2021).

\section{Discussão}

\section{Categoria I: Protocolos de atendimento inicial, estimativa de Superfície Corporal Queimada e analgesia.}

Da análise dos artigos selecionados, percebe-se um consenso acerca da importância da definição de um protocolo de atendimento ao queimado, levando-se em conta também diferenças na conduta em populações especiais, como gestantes e crianças (A3 e A7 respectivamente).

Nesse contexto, A2 discorre acerca dos benefícios da padronização do atendimento desde a conduta inicial, resultando em uma otimização do serviço através da organização e planejamento dos cuidados, influenciando positivamente no prognóstico do paciente, enquanto que A1 deixa claro sobre a existência de controvérsias acerca das condutas adotadas pela equipe médica, e destaca que os cuidados pré-hospitalares e iniciais do paciente são necessários para um bom prognóstico. A obra A6 atenta para a importância do trabalho multiprofissional no seguimento do grande queimado, o que resulta em redução de mortalidade, embora ocasione uma maior demanda do sistema de saúde.

No que diz respeito ao atendimento inicial, além da atenção dada à remoção da fonte de calor, roupas e adornos, é imprescindível o resfriamento imediato da lesão com água fria corrente entre 10 minutos e uma hora e início em até 3 horas como prega A1. Entretanto, a equipe de atendimento deve ser cuidadosa ao promover o resfriamento de lesões extensas por conta do risco de hipotermia associado, sendo recomendada a irrigação do local com aquecimento corporal concomitante.

Ainda no atendimento inicial, o cálculo da extensão das queimaduras é essencial para auxiliar na determinação do grau de comprometimento do paciente, além de conduzir tomadas de decisão, como citado em A8. Para isso, pode-se fazer o cálculo da Superfície Corporal Queimada (SCQ) a partir da Regra dos 9, criada por Wallace e Pulaski, em que o corpo humano é dividido em regiões anatômicas que representam porcentagens de 9 ou múltiplos de 9 da superfície corporal total (Brasil, 2012; Pechara et al., 2020).

Ao tratar sobre a analgesia a ser aplicada em pacientes queimados, é importante entender que as queimaduras de segundo grau são as maiores causadoras imediatas de dor no paciente, já que os ferimentos em terceiro grau lesam também o tecido nervoso, afetando a nocicepção (A8). Nesse contexto, esta revisão aponta que o medicamento mais utilizado para 
analgesia é a morfina, que é ajustada às necessidades de cada paciente, sempre de modo a evitar a administração de altas doses e prevenir a ocorrência de efeitos adversos. Além dela, a literatura traz que outras drogas podem ser utilizadas para auxiliar no controle da dor, como fentanil e dipirona (A8).

Outra conduta importante no tratamento do paciente queimado é a reposição volêmica. Há urgência na reidratação parenteral em queimaduras graves, uma vez que, uma demora em realizar esse procedimento aumenta a chance de desenvolvimento de choque hipovolêmico. É utilizada pelos profissionais a fórmula de Parkland (4 $\mathrm{ml}$ x $\mathrm{kg}$ x SCQ) para calcular a reposição eletrolítica a ser administrada. Esse cálculo se baseia na massa corporal e na superfície queimada e ajuda também a evitar a hiperidratação (A8). Em A8, há a recomendação de se utilizar nessa conduta o soro ringer lactato e, durante a sua administração, ajustar o volume de acordo com as condições clínicas do paciente, como ritmo diurético e pressão arterial média.

Devido à complexidade no tratamento de pacientes em quadro grave, A2 fala sobre a importância da existência de Centros ou Unidades de Tratamento de Queimados para permitir um trabalho intensivo e direcionado a esses pacientes, exercido por uma equipe multiprofissional. Com relação ao exposto, o tratamento moderno de queimaduras é baseado na excisão do tecido queimado e aplicação precoce de enxerto, acompanhado de reabilitação agressiva (A6). Os cuidados devem ser seguidos pelos pacientes do início ao fim, haja vista que, dessa forma, há uma maior chance de evitar sequelas funcionais. Esse fato é comprovado pela pesquisa apresentada em A6, que revelou que dos pacientes que apresentaram sequelas, 90\% não haviam seguido o tratamento da forma correta; e somente $10 \%$ apresentaram cicatrização resistente ao tratamento.

\section{Categoria II: Populações especiais}

Como mencionado no início da discussão, a definição de protocolo diferenciado, atendendo às necessidades de crianças e gestantes - populações especiais - é de extrema importância para um melhor prognóstico desses pacientes. Nesse contexto, A4 faz análise do tipo de lesão mais comum em crianças, as escaldaduras, causadas pelo contato da pele com líquidos em altas temperaturas e capazes de gerar lesões debilitantes de terceiro grau. De acordo com estudo realizado por Takino et al. (2016), o qual analisou a epidemiologia de crianças e adolescentes vítimas de queimaduras admitidos em centro de tratamento de queimados concluiu-se que, dos 177 prontuários analisados, o principal agente da queimadura foi escaldadura, em 53\% dos casos, seguido de fogo em $30 \%$ e contato com sólido aquecido em $10 \%$. Quanto ao atendimento de gestantes queimadas, além de estimar a incidência mundial de queimaduras em gestantes (3\% a 7\%), A3 discorre sobre as complicações mais comuns para o feto: sofrimento fetal, aborto e trabalho de parto prematuro. Nesse contexto, a pesquisa de Parikh et al (2015) indica a cesárea de urgência em gestantes com fetos viáveis e que apresentam mais de 55\% de SCQ a fim de melhorar o prognóstico materno-fetal. Por isso, o tratamento da gestante queimada está intimamente relacionado com a sobrevivência da mãe e do feto.

\section{Categoria III: Lesões por descargas elétricas e prevenção.}

Tratando-se da importância do conhecimento sobre o mecanismo causador da lesão, é necessário citar as queimaduras causadas por descargas elétricas, tratadas em A5, que chamam a atenção por exigirem cuidados, recursos e conhecimentos distintos. Nota-se que, mesmo raras, lesões por alta tensão têm a maior porcentagem de amputações dentre todos os tipos de queimaduras uma vez que acometem partes moles, neuromusculares e podem causar tetania muscular, fatores que conferem grave morbidade a esse tipo de queimadura. Ademais, nesses casos não é possível confiar no cálculo da SCQ, já que este subestima a extensão real da injúria, pois faz análise somente da superfície cutânea acometida, enquanto que descargas elétricas afetam principalmente tecidos mais profundos.

Os traumas de queimaduras geram um gasto anual muito alto ao sistema público de saúde, dado que em média 100.000 brasileiros são hospitalizados por ano vítimas de queimaduras (A1). Nessa perspectiva, surge a necessidade de 
prevenir esses traumas, considerando que a maioria deles ocorrem de forma acidental e que os mais suscetíveis a eles são idosos e crianças (A1). É um consenso na literatura utilizada que as formas mais eficazes de prevenção são: estratégias de educação e conscientização para um manuseio adequado de fatores potenciais a acidentes, a exemplo água quente e produtos químicos.

O presente estudo apresenta limitações no que diz respeito aos critérios utilizados para seleção dos artigos, como o uso dos descritores "Queimados" "Unidades de queimados" e "Primeiros socorros" que tiveram a finalidade de filtrar dados com informações relevantes à proposta do texto. Ainda, percebeu-se a deficiência em número de trabalhos recentes relacionados ao tema em questão nas bases de dados utilizadas para coleta, o que restringiu significativamente a quantidade de conteúdo disponível para leitura. Como pontos positivos, observa-se que foram usados três idiomas para busca, português, inglês e espanhol, fato que amplia o acesso à bibliografia pertinente. Além disso, como contribuição para a literatura científica, foi feito um apanhado sobre o atendimento e manejo geral de pacientes queimados, mas também uma pesquisa ampla sobre as peculiaridades desse atendimento nas populações especiais e os diferentes mecanismos de lesão térmica.

\section{Conclusão}

O atendimento pré-hospitalar ao paciente acometido por queimaduras exige avaliação clínica, habilidades e conhecimentos intrínsecos sobre primeiros socorros para a otimização do prognóstico dessa injúria. A partir dos resultados do estudo, foi possível identificar alguns temas discutidos pelos autores, tais como coberturas utilizadas nas áreas queimadas, retirada de adornos e processo de triagem, sendo medidas pré-hospitalares, como as citadas, imprescindíveis para um bom prognóstico dos pacientes vítimas de queimaduras.

Além disso, concluiu-se que o estabelecimento de um protocolo para atendimento e tratamento dos pacientes vítimas de queimaduras, independente do grau da queimadura ou da particularidade do paciente, a qual inclui-se crianças e gestantes, mostra-se efetivo no intuito de promover resultados favoráveis na recuperação dos pacientes queimados, sendo eficaz na redução de variáveis de morbidade e no quadro geral dos acometidos. Ademais, destaca-se a importância da padronização do atendimento em um contexto mais amplo, pois dessa forma seria possível a obtenção de resultados otimizados, diante de uma convenção de medidas embasadas cientificamente, cabendo a cada instituição de saúde, dentro da sua complexidade e especificidades, estabelecer os protocolos e rotinas técnicas de atendimento ao paciente queimado.

A partir dos estudos analisados ainda foi possível identificar que a vítima de queimadura representa um paciente desafiador em nível médico, psicológico e social, que deve ser tratado meticulosamente, da admissão à reabilitação e posterior reinserção social. Assim, vale ressaltar que o trabalho multidisciplinar apresenta-se como pilar fundamental no tratamento e consequentemente na prevenção de sequelas funcionais.

Evidencia-se que pacientes que requerem atendimento ainda mais singular, como crianças e gestantes, continuam como grupo que merecem atenção especial, já que nas crianças, por exemplo, às queimaduras, principalmente as lesões por escaldadura, continuam a ser um acometimento comum com potencial para consequências devastadoras ao longo da vida, apesar das campanhas de prevenção. Nas gestantes, deve ser concedida atenção singular às pacientes de primeiro e terceiro trimestre. As primeiras merecem foco quanto às medicações que serão utilizadas durante o seu tratamento, devido ao risco de teratogenicidade. Nas pacientes de terceiro trimestre, passa a ter maior importância a monitorização fetal com a cardiotocografia devido ao risco de sofrimento fetal, abortamento e parto prematuro.

\section{Referências}

Ambrosoni, M., Telechea, H., Cristiani, F., Manaro, B., Pizarro, M., \& Menchaca, A. (2018). Propuesta de tratamiento del gran quemado en la unidad de cuidados intensivos del CHPR. Arch. Pediatr. Urug., 89 (2):129-134. 
Bezerra, A. F. C., Galvão, P. V. M., Conceição, J. M., Silva, J. M. N., Souza, E. H. A., \& Andrade, E. S. S. (2020). Mortalidade devido a queimaduras em crianças entre zero e quatro anos no Brasil. Brazilian Journal of Development, 6 (7): 43012-43023.

Brasil. Ministério da Saúde. (2012). Cartilha para tratamento de emergência das queimaduras.

Brasil. Ministério da Saúde. Portal da Saúde. (2017). Queimados [Internet]. https://www.saude.gov.br/component/content/article/842-queimados/40990-.

Cantarelli-kantorski, K. J., Martins, C. L., Andolhe, C., Brum, A. L., Dal Pai, D., \& Echevarría-Guanilo, M. E. (2014). Caracterização dos atendimentos por queimaduras em um serviço de pronto-socorro. Rev Bras Queimaduras, 13(1): 38-43.

Faroni, N., Coria, F., Tonegusso, J, Lucía, R. V., Levy, R., \& Taljame, M. L. (2018). Manejo interdisciplinario de pacientes quemados: pilar fundamental para la reducción de secuelas funcionales. Rev.med rosário, 1(84):26-29.

Gilbert, A. D., Rajha, E., El Khuri, C., Bou Chebl, R., Mailhac, A., Makki, M., \& El Sayed, M. (2017). Epidemiology of burn patients presenting to a tertiary hospital emergency department in Lebanon. Burns, 44(1):218-25.

Jamshidi, R., \& Sato, T. T. (2016). Initial Assessment and Management of Thermal Burn Injuries in Children. ped. in rev, 34 (395): 395-404.

Kim, L. K., Martin, H. C., \& Holland, A.J. (2012). Medical management of paediatric burn injuries: best practice. J Paediatr Child Health, 48(4):290-295.

Lee, D. H., Desai, M. J., \& Gauger, E. M. (2019). Electrical Injuries of the Hand and Upper Extremity. J Am Acad Orthop Surg, $27(1)$ :e1-e8.

Leite, J. R. N. F., Fernandes, E. L., Takassi, G. F., Oliveira, A. F., \& Ferreira, L. M. (2018). Gestação e queimadura: experiência de unidade de queimaduras em Hospital Universitário. Rev. Bras. Cir. Plást., 33(3):423-427

Lima, L. S., Correia, V. O. S., Nascimento, T. K. G., Chaves, B. J. P., Silva, J. R. S., Alves, J. A. B., Dantas, D. V., \& Ribeiro, M. C. O. (2017). Profile of Burn Victims Attended by an Emergency Unit. Int Arch Med., 10(10):1-9.

Moraes, L. P., Echevarría-Guanilo, M. E., Martins C. L., Longaray, T. M., Nascimento, L., Braz, D. L., Sebold, L. C., \& Antoniolli, L. (2016). Apoio social e qualidade de vida na perspectiva de pessoas que sofreram queimaduras. Rev Bras Queimaduras,15(3):142-7.

Moraes, R. Z. C., Frempong, R. F. R., Freitas, M. S., Soares, A. U. S., Pereira, R. O., Carvalho, G. G, Macedo, F. N., Borges, K. S., \& Cintra, B. B. (2014). Análise comparativa da morbimortalidade antes e após implantação de protocolo de atendimento ao queimado. Rev Bras Queimaduras, 13(3):142-146.

Moulin, L. L., Dantas, R. A. N., Aiquoc, K. M, Dantas, D. V., Vasconcelos, E. F. L., Lima, K. R. B., \& Lima, Maria S. M. (2018). Perfil sociodemográfico e clínico de vítimas de queimaduras atendidas em um hospital de referência. Revista Nursing, 21(238): 2058-2062.

Parikh, P., Sunesara, I., Lutz, E., Kolb, J., Sawardecker, S., \& Martin, J. N. J. (2015). Burns during pregnancy: implications for maternal-perinatal providers and guidelines for practice. Obstet Gynecol Surv., 70(10):633-643.

Pechara, B. B. (2019). O paciente queimado: um panorama e perspectivas terapêuticas. Repositório de Trabalho de Conclusão de Curso, Centro Universitário UNIFACIG, Manhuaçu.

Rocha, N. M., Silva, E. Ângelo da, Silva, E. M., Melo, C. J. R. de, \& Mota, L. M. (2020). Atendimento inicial às vítimas de queimaduras: uma revisão de integrativa. Caderno De Graduação - Ciências Biológicas E Da Saúde - UNIT - ALAGOAS, 6(1): 11-20.

Takino, M. A., Valenciano P. J., Itakussu E. Y., Kakitsuka E. E., Hoshimo A. A., Trelha C. S., \& Fujisawa, D. S. (2016). Perfil epidemiológico de crianças e adolescentes vítimas de queimaduras admitidos em centro de tratamento de queimados. Rev Bras Queimaduras, 5(2):74-79.

Valente, T. M., Nascimento, M. F. A., Silva Júnior, F. R., Souza, J. P. F., Martins, C. B, Valente, T. M., Brito, M. E. M, \& Brito, M. E. M. (2018). Importância de um atendimento pré-hospitalar efetivo a adultos vítimas de queimaduras: uma revisão integrativa. Rev Bras Queimaduras, 17(1):50-55. 